\title{
The Motion of Interplanetary Dust Particles
}

\author{
I.R. East and N.K. Reay \\ Astronomy Group, Department of Physics, Blackett Laboratory, \\ Imperial College, Prince Consort Road, \\ LONDON SW7 2BZ \\ ENGLAND
}

\begin{abstract}
Radial velocity measurements on the solar MgI 5183.618 Fraunhofer absorption line in the Zodiacal Light spectrum have been made with a microprocessor-controlled servo-stabilised Fabry-Perot interferometer. Observations were made at 5 and 10 degree intervals in the ecliptic plane between morning and evening elongations of 25 degrees. These new data are of much greater precision and coverage than any previously obtained. Obtained over a two year period, the observations show a consistent evening/morning asymmetry in the radial velocity curve with the Gegenschein receding from the Earth at $4 \mathrm{Km} / \mathrm{sec}$. These data do not support the hypothesis that the majority of interplanetary dust grains are in hyperbolic trajectories.
\end{abstract}

\section{THE INSTRUMENT}

Because the Zodiacal Light is a very faint source it is necessary to use an interferometer of high luminosity. When only a narrow spectral region is to be observed the Fabry-Perot etalon offers a gain in luminosity over other types of instrument.

The MgI 5183.6A Fraunhofer line was chosen because of it's depth, cleanness, freedom from blending and from contamination by airglow, geocoronal or meteoric emmission. It has been used with some success on previous occasions, (Hicks, May and Reay, 1974; James \& Smeethe, 1970).

An etalon of diameter $80 \mathrm{~mm}$ and Finesse -24 with a plate separation of $87 \mu \mathrm{m}$ was employed giving a Free spectral Range of 14. 4月.The etalon gap and paralellism were servo-stabilised using the technique of capacitance micrometry (Hicks, Reay and Scadden, 1974). Servo actuation is by piezo-electric transducers. An $80 \mathrm{~mm}$ diameter 3 cavity interference filter was used as prefilter. Residual drift of the etalon plate spacing was less than $\lambda / 500$ corresponding to a spectral drift of $0.03 \AA$ per hour. Periodic calibration scans of a cold Magnesium hollow cathode lamp ensured that systematic errors due to wavelength drift were always less than $0.01 \AA$.

The photomultiplier employed was a RCA31043A-02 with a 81

R. H. Giese and P. Lamy (eds.), Properties and Interactions of Interplanetary Dust, 81-84.

(C) 1985 by D. Reidel Publishing Company. 
gallium-arsenide photocathode which, when cooled to -70 degrees $C$, gave a dark count of between 1 and $4 \mathrm{~Hz}$.

\section{OBSERVATIONS AND DATA REDUCTION}

The site chosen was the one used by Hicks et al (1974), Izana on Tenerife in the Canary Islands. Observing trips were made in March, July and September in 1981 and 1982 and also in March 1983. Two further trips in July and September 1983 were unfortunately both wiped out by bad weather. This still gave a total of more than 60 nights observing.

A spectral range of $\sim 6 \AA$ centred on the $\mathrm{MgI} 5183.6 \AA$ absorption line was scanned at intervals of $0.25 \AA$. Scans accumulated over several nights at elongation intervals of 5 or 10 degrees were co-added to improve signal to noise ratio. The summed scans were then cross-correlated with a gaussian profile of equal width (and similar shape) to that of the unshifted line when convolved with the instrumental profile. The peak of the cross-correlation function is then used to indicate the position and thus the shift of the line.

It is important to note that any small component of the Zodiacal Light originating from dust moving with velocities greater than about $50 \mathrm{~km} / \mathrm{sec}$ will not affect the position of the peak of the cross-correlation function as much as it would the first moment (or centre of gravity).

\section{RESULTS}

Figure 1 shows the sum total of the Doppler-shift results gathered over the whole observing program. The salient points to note are;

i) the red-shift of the Gegenschein,

ii) asymmetry between evening and morning,

iii) that the spread of the points is consistent with the calculated standard errors.

Figure 1 Measured Dopplershift plotted as a function of elongation.

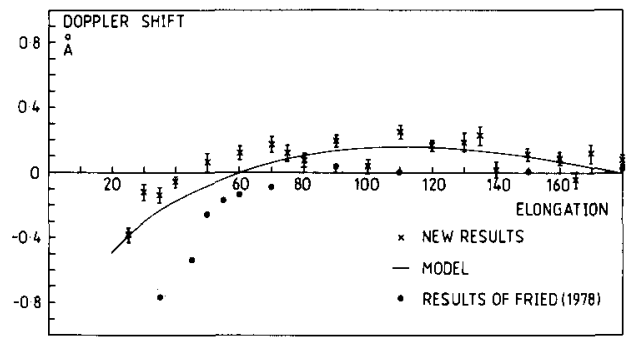

Note that the error at the Gegenschein is much less than elsewhere due to the deliberately applied priority to this point. Thus the red-shift of the Gegenschein is more significantly determined than the nature of the asymmetry.

Figure 2 shows the new results folded about the anti-solar point to ease comparison with the data from Fried (1978) (averaged for each elongation) and with a simple model in which the dust is in circular 
orbits, unaffected by radiation pressure, with a radial distribution of number density of the form;

$$
n(r)=K / r^{1 \cdot 3}
$$

determined from Helios and Pioneer spacecraft, (Link et al., 1976). Diffraction effects are neglected, since these are only important for elongations below 20 degrees, and isotropic scattering is assumed.

Figure 2. The new results folded about the antisolar point to facilitate comparison with the data of Fried and a simple model of circular Keplerian orbits.

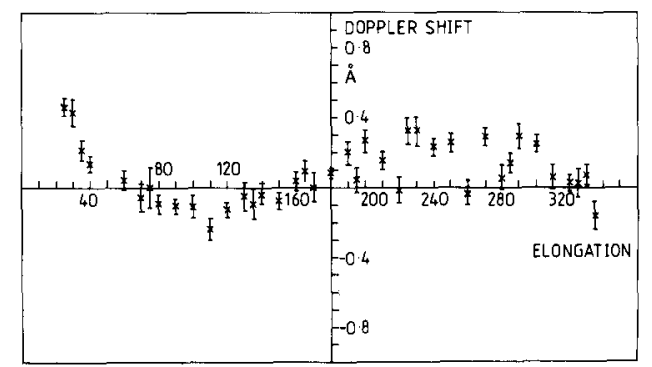

This model is obviously not wholly inadequate. However it is inherently incapable of explaining the observed asymmetry or the recession of the Gegenschein. (The former is of course averaged out by the folding process).

\section{INTERPRETATION OF DOPPLER-SHIFT DATA}

\subsection{Elongations less than 180 degrees}

It may be shown that, in the expression for the observed Doppler-shift along a line of sight corresponding to a particular elongation, there exists a single factor $x$ which accounts for any deviations the dust may exhibit from circular Keplerian orbits, (East, 1984). It mayalso be shown also that $\chi$ factorises into three components, one each for the effect of radiation pressure, orbital eccentricity and orbital inclination provided radial invariance is assumed for optical properties, size distribution and distribution of orbital parameters. With the help of the Haug integral (Haug, 1958), these three factors are readily derived, (East, 1984).

Note that the effects of radiation pressure, orbital eccentricity and orbital inclination on Doppler-shifts measured in the ecliptic plane are indistinguishable. Also the measured values of $x$ as a function of $\varepsilon$ may be used as a probe of radial invariance.

\subsection{The Gegenschein}

It may be shown (East, 1984) that to observe a red-shift in the Gegenschein implies directly the existence of a component within the Zodiacal dust cloud of particles in hyperbolic trajectory, i.e unbound to the solar system which are contributing to the observed Zodiacal Light.

Because the reduction technique employed is insensitive to any 
structure in the wings of the line the measured Doppler-shifts will be unaffected by a component with line of sight velocities near or in excess of the velocity of escape from the solar system at 1 A.U..

The fact that a shift in the Gegenschein has been observed can only be interpreted as implying that the particles originate at solar distances which are NOT $\ll 1$ A.U. .

\section{CONCLUSIONS}

The weighted mean value of $x$ is between $0.85+/-0.04$ and $0.89+1-0.04$ depending only on choice of scattering function (observed VSF or isotropic) and radial distribution index (1.0 to 1.5$)$ employed as the model.

There clearly exists a component of the Zodiacal dust cloud escaping from the solar system on hyperbolic trajectories which does contribute observably to the zodiacal light. It is possible that this contribution is seasonally variable and that the observed recession of the Gegenschein is only a fairly coarse average value.

To be consistent with a red-shift being observed at all, the escaping particles must originate at solar distances which are not $\ll 1$ A.U.. This is consistent with conclusions reached by Whipple (1976) based on the model by Zook \& Berg (1975) of submicron escaping particles as collisional debris.

ACKNOWLEDGEMENTS: The authors wish to thank the Instituto del Astrofisica de Canaries for use of site and facilities at the Observatorio de Izana, Tenerife.

One of us, (I.R. East), gratefully acknowledges receipt of an. S.E.R.C. studentship during the greater part of this research.

The authors are indebted to Professor David Beard of the University of Kansas for many helpful and useful discussions.

\section{REFERENCES}

East I.R.: 1984, in preparation

East I.R. \& Reay N.K.: 1984, submitted to Astron. \& Astrophys. March 1984

Fried J.W.: 1978, Astr. \& Astrophys., 68, 259

Haug U.: 1958, Z. Astrophys., 44,71

Hicks T.R., May B., Reay N.K.: 1974, Mon. Not. R. Astr. Soc., 166,439

Hicks T.R., Reay N.K., Scadden R.J.: 1974, J. Phys. E. (Sci. Instr.) 7,27

James J.F. Smeethe M.J.: 1970, Nature, 227, 589

Link H., Leinert C., Pitz E., Salm N.: 1976, Lect. Notes in Phys., 48, 19

Whipple F.L.: 1976, Lect. Notes in Phys., 48, 403

Zook H., Berg O.E.: 1975, Planet.Spa. Sci., 23, 183 